\title{
SINGULARITIES OF SPACES OF FLAT BUNDLES OVER COMPLEX MANIFOLDS
}

\author{
BY
}

B. WONG

\begin{abstract}
We use Fox differential calculus on free group to study the singularities of complex analytic varieties arising from flat bundles over complex manifolds. Criteria of regularity in terms of cohomology of the fundamental group of the underlying manifold are established.
\end{abstract}

1. Introduction and statement of results. Given a finitely presented group $Q=\left\{R_{1}, R_{2}, \ldots, R_{L}: x_{1}, x_{2}, \ldots, x_{k}\right\}$ with $L$-relations and $k$-generators, $G$ a finite dimensional complex Lie group, and $S=\operatorname{Hom}(Q, G)$ the family of all representations of $Q$ into $G$, we can identify $S$ with a complex analytic variety in $G \times G \times \cdots \times G$ ( $k$ times); as we can $\operatorname{regard}\{S=\operatorname{Hom}(Q, G)\}$ as the locus described by the system of equations $\left\{R_{1}=0, R_{2}=0, \ldots, R_{L}\right.$ $=0$. .

Our basic problem is to determine what type of representations will give a regular point of the analytic variety $S$. It is a group theoretical problem, and we shall give a criterion of the singularities of $S$ in terms of the cohomology of the finitely presented group $Q$. One of the main contributions of this paper is to develop the Fox differential calculus on free groups to treat the singularities of $S$ systematically. Theoretically this method can be applied to any finitely presented group. In this way one can obtain the results of [5] and [2(4)] of Riemann surfaces as a special case of our main theorem.

Before stating our results, we are going to give some definitions.

Definition. $Q$ is said to be of finite type if it admits a finite resolution of a $Z Q$-module $A$.

We shall denote the Euler characteristic of $Q$ by the symbol of $X(Q)$.

(For detail one can consult [1], also §3(2).)

Let $g$ be the Lie algebra of $G$, and ad $\rho$ the adjoint representation of $Q$ into the $\operatorname{End}(g)$ with respect to a given representation $\rho$ of $Q$ into $G$. The symbol $H^{i}(Q, \operatorname{ad} \rho)$ will denote the $i$ th cohomology of $Q$ with coefficients given by the adjoint representation ad $\rho$ of $Q$ in the Lie algebra $g$.

THEOREM. Let $Q$ be any finitely presented group.

Received by the editors February 15, 1976.

AMS (MOS) subject classifications (1970). Primary 32C40; Secondary 32L05, 32 G13.

- American Mathematical Society 1977 
(A) If $Q$ is of finite type, then

$$
\begin{aligned}
\rho \in S \text { is regular } \leftrightarrow & \sum_{i \geqslant 2}(-1)^{i} \operatorname{dim}_{C} H^{i}(Q, \operatorname{ad} \rho) \\
& =h_{\rho}+(X(Q)-1) \cdot \operatorname{dim}_{C} G .
\end{aligned}
$$

(B) If $Q$ is just any finitely presented group, then

$$
\begin{aligned}
\rho \in S \text { is regular } \leftrightarrow & \operatorname{dim}_{C} H^{0}(Q, \operatorname{ad} \rho)-\operatorname{dim}_{C} H^{1}(Q, \operatorname{ad} \rho) \\
& =\operatorname{dim}_{C} G-h_{\rho} .
\end{aligned}
$$

REMARK. Finitely presented group of finite type is of particular interest. Applications of our theorem will be given later. We suspect that there might be a natural interpretation of the structure of the singularities of $S$ in terms of the higher cohomology of $Q$.

$h_{\rho}$ is the height of the maximal ideal of the local ring of the complex analytic variety $S$ at the point $\rho$. (See [2(2)] for the basic definition.)

Definition. A word $R$ containing $\left\{x_{1}, x_{2}, \ldots, x_{k}\right\}$ is reduced if it does not contain adjacent symbols $x_{i}$ and $x_{i}^{-1}$. It will be called cyclically reduced if its first and last symbols are not $x_{i}$ and $x_{i}^{-1}$.

A word is called irreducible iff it is not a proper power of some other word, that is $R=M^{m}, m>1, M$ is another word containing $\left\{x_{1}, x_{2}, \ldots, x_{k}\right\}$.

COROLlary. Let $G$ be any finite dimensional complex Lie group as before, $Q=\left\{R_{1}, R_{2}, \ldots, R_{L}: x_{1}, x_{2}, \ldots, x_{L+s}\right\}$, where each $R_{i}$ is irreducible and cyclically reduced, and, for each $t x_{t}$ and $x_{t+s}$ are the first and last of the $x_{i}$ that occur (in the order of the subscripts). Then

$$
\rho \in S \text { is regular } \leftrightarrow \operatorname{dim}_{C} H^{2}(Q, \operatorname{ad} \rho)=h_{\rho}-s \cdot \operatorname{dim}_{C} G .
$$

REMARKs. (1) Examples of the above corollary can be found in knot groups.

(2) The following known results of [5] and [2(4)] can be derived from this corollary.

If $G=\mathrm{SL}(n, C), Q$ is the fundamental group of compact Riemann surface of genus $\geqslant 2$, then

$$
\rho \in S \text { is regular } \leftrightarrow \rho \text { has only scalar commutants. }
$$

If $G=U(n), Q$ is the fundamental group of compact Riemann surface of genus $\geqslant 2$, then

$$
\rho \in S \text { is regular } \leftrightarrow \rho \text { is an irreducible representation. }
$$

These two results are important in studying the moduli of flat bundles over compact Riemann surfaces. 
2. A basic lemma. The basic lemma given here is essentially a generalization of [5].

Let $Q=\left\{R_{1}, R_{2}, \ldots, R_{L}: x_{1}, x_{2}, \ldots, x_{k}\right\}$ be a finitely presented group with $L$-generators and $k$-generators. We can consider $R=\left(R_{1}, R_{2}, \ldots, R_{L}\right)$ as a mapping from $G^{k}$ to $G^{L}$. In this way one can identify $S$ with the set $R^{-1}(1)$ (where 1 is the identity element of $G^{L}$ ). As usual the differential of $R$, denoted by $d R$, can be considered as a linear mapping between the tangent spaces of $G^{k}$ and $G^{L}$. In the sequel $Z^{1}(Q, \operatorname{ad} \rho)$ will be the one cocylce of $Q$ with respect to the adjoint representation ad $\rho$ into $\operatorname{End}(g)$.

LEMMA 1. Kernel of $(d R)_{\rho}=Z^{1}(Q$, ad $\rho)$ for all $\rho \in S$.

Proof. Let $A=G \times G \times G \times \cdots \times G$ ( $k$ times), $B=G \times G \times G \times \cdots$ $\times G$ ( $L$ times), $A_{\rho}=$ tangent space of $A$ at $\rho$, where $\rho \in S$. The differential $(d R)_{\rho}: A_{\rho} \rightarrow B_{1}$ is a linear mapping between $A_{\rho}$ and $B_{1}$, where $B_{1}=$ tangent space of $B$ at 1 . We want to show the kernel of $(d R)_{\rho}$ can be identified with $Z^{1}(Q$, ad $\rho)$ in a natural manner.

For this purpose, we let $w=d \bar{B} \cdot \bar{B}^{-1}$ be the right-invariant MaurerCartan form of $\bar{B}$; then $d R \cdot R^{-1}$ is the inverse image of $w$ by $R$, i.e. $d R \cdot R^{-1}=R^{*}(w)$, and so the kernel of $(d R)_{\rho}$ is exactly $\left\{v \in A_{\rho} \mid d R \cdot R^{-1}(v)\right.$ $=0\}$. Let the generators of $Q$ be $\left\{x_{1}, x_{2}, \ldots, x_{k}\right\}$. A tangent vector $v$ to $A$ can be identified (by means of right translation) with $v=\left(A_{1}, A_{2}, \ldots, A_{k}\right)$. In the following we shall denote $F$ to be the free group with generators $\left\{x_{1}, x_{2}, \ldots\right.$, $\left.x_{k}\right\}$.

The following theorem is known.

THEOREM. Let $F$ be either the free monoid or the free group generated by a set $K$ of symbols, and $H$ a F-module. Suppose that an arbitrary mapping $f: K \rightarrow H$ is given; then there exists one and only one extension of $f$ to one cocycle of $F$ into $H$.

An element of $Z^{1}(F, \operatorname{ad} \tilde{\rho})$ is a mapping $t: F \rightarrow g$ such that $\forall \gamma_{1}, \gamma_{2} \in F$ satisfying the following equation $(\tilde{\rho} \in A)$

$$
t\left(\gamma_{1} \cdot \gamma_{2}\right)=\left(\operatorname{ad} \tilde{\rho}\left(\gamma_{1}\right)\right) \cdot t\left(\gamma_{2}\right)+t\left(\gamma_{1}\right)
$$

Applying this theorem to our case, associated to each tangent $v=\left(A_{1}\right.$, $\left.A_{2}, \ldots, A_{k}\right)$ at $\tilde{\rho}$ there is a unique 1-cocylce $s_{v}$ of $F$ with respect to the adjoint representation ad $\tilde{\rho}$ with $s_{v}\left(x_{i}\right)=A_{i}$ for each $i$ corresponding to each particu$\operatorname{lar} v, \tilde{\rho} \in A$.

We claim that $d R \cdot R^{-1}(v)=s_{v}\left(R_{i}\right)$ for all $i$.

(REMARK. One has to note that $p$ is a 1-cocycle of $Q$ iff $p$ is a 1-cocycle of $F$ and $p\left(R_{i}\right)=0$ for all $i$.)

To prove our claim, consider $A \times F$ as an analytic manifold, where $F$ can 
be regarded a discrete manifold. For each point of $A$ one can define a representation of $F$ into $G$. Therefore we can construct an analytic map $\Phi: A \times F \rightarrow G,(\tilde{\rho}, \gamma)=\tilde{\rho}(\gamma)$, where $\tilde{\rho} \in A, \gamma \in F$.

If $v \in A_{\tilde{\rho}}$, then $d \Phi \cdot \Phi^{-1}(v)$ is a function $\psi$ on $F$ with values in $g ; \psi$ is a 1 cocycle for ad $\tilde{\rho}$.

Actually for each $X \in A, \gamma_{1}, \gamma_{2} \in F$, it is obvious that

$$
\Phi\left(X, \gamma_{1} \cdot \gamma_{2}\right)=\Phi\left(X, \gamma_{1}\right) \cdot \Phi\left(X, \gamma_{2}\right)
$$

then we have

$$
\begin{aligned}
d \Phi\left(X, \gamma_{1} \cdot \gamma_{2}\right)= & \Phi\left(X, \gamma_{1}\right) \cdot d \Phi\left(X, \gamma_{2}\right)+d \Phi\left(X, \gamma_{1}\right) \cdot \Phi\left(X, \gamma_{2}\right) \\
d \Phi\left(X, \gamma_{1} \cdot \gamma_{2}\right) \cdot \Phi\left(X, \gamma_{1} \cdot \gamma_{2}\right)^{-1}= & d \Phi\left(X, \gamma_{1} \cdot \gamma_{2}\right) \Phi\left(X, \gamma_{2}\right)^{-1} \cdot \Phi\left(X, \gamma_{1}\right)^{-1} \\
= & {\left[\Phi\left(X, \gamma_{1}\right) \cdot d \Phi\left(X, \gamma_{2}\right)+d \Phi\left(X, \gamma_{1}\right) \cdot \Phi\left(X, \gamma_{2}\right)\right] } \\
& \cdot \Phi\left(X, \gamma_{2}\right)^{-1} \cdot \Phi\left(X, \gamma_{1}\right)^{-1} \\
= & \Phi\left(X, \gamma_{1}\right)\left[d \Phi\left(X, \gamma_{2}\right) \cdot \Phi\left(X, \gamma_{2}\right)^{-1}\right] \Phi\left(X, \gamma_{1}\right)^{-1} \\
& +d \Phi\left(X, \gamma_{1}\right) \cdot \Phi\left(X, \gamma_{1}\right)^{-1} .
\end{aligned}
$$

Hence it is equivalent to say

$$
\psi\left(\gamma_{1} \cdot \gamma_{2}\right)=\tilde{\rho}\left(\gamma_{1}\right) \cdot \psi\left(\gamma_{2}\right) \cdot \tilde{\rho}\left(\gamma_{1}\right)^{-1}+\psi\left(\gamma_{1}\right) \quad \forall \gamma_{1}, \gamma_{2} \in F .
$$

That is the same to say $\psi$ is a 1-cocycle for ad $\tilde{\rho}$.

Hence we come to the conclusion that $\psi=s_{v}$.

Now for each $R_{i}$ of $R=\left\{R_{1}, R_{2}, \ldots, R_{L}\right\}$, we have by definition

$$
\Phi\left(X, R_{i}\right)=R_{i}(X), \quad \forall 1 \leqslant i \leqslant L, \forall X \in A,
$$

and we can regard each $R_{i}$ as a mapping from $G^{k}$ to $G$ so we have

$$
s_{v}\left(R_{i}\right)=\psi\left(R_{i}\right)=d R_{i} \cdot R_{i}^{-1}(v), \quad i=1,2, \ldots, L .
$$

It follows immediately that $v$ belongs to the kernel of $d R_{i}$ iff the 1-cocycle $s_{v}$ (on $F$ ) is zero on $R_{i}$. However, it is obvious that the kernel of $d R \cdot R^{-1}$ is equal to the intersection of the kernel of $d R_{i} \cdot R_{i}^{-1}: i=1,2, \ldots, L$.

This finishes the proof of the claim.

Together with the remark on p. 453 our proof of the lemma is therefore completed.

The following fact is well known. (See [2(2)] for example.)

THEOREM. (A) The imbedded dimension of a germ $S$ of an analytic variety is the minimal number of generators of the maximal ideal $M_{S}$ of the local ring $O_{S}$. 
(B) For any germ $S$ of an analytic variety

$$
\text { imbed } \operatorname{dim} S=\operatorname{dim}_{C}\left(M_{S} /\left(M_{S}\right)^{2}\right) \text {. }
$$

An immediate observation is that a point at $\rho \in S$ is regular if and only if $(\operatorname{dim} S$ at $\rho)=h_{\rho}=\operatorname{dim}_{C}\left(\left(M_{S}\right)_{\rho} /\left(M_{S}\right)_{\rho}^{2}\right)$.

Now we can come back to our problem. In our case $S$ is an imbedded subvariety of $G^{k}$. Let the dimension of $G^{k}$ be $n$, and the dimension of $G^{L}$ be $m$. Consider $R: G^{k} \rightarrow G^{L}$ as mapping between complex manifolds. As before $R=\left(R_{1}, R_{2}, \ldots, R_{L}\right)$, and $\left(\partial R_{i} / \partial X_{j}\right)_{\rho}$ is the Jacobian matrix of $R$ at $\rho \in S$ (with respect to the local coordinates $\{X\}$ ).

Suppose that $r$ is the rank of the Jacobian of $R$ at $\rho$; we have $\operatorname{dim}_{C} \operatorname{ker}(d R)_{\rho}$ $=n-r$. Let $f_{1}, f_{2}, \ldots, f_{w}$ be the generators of the ideal sheaf of the complex analytic variety $S$ at $\rho$. The subspace of $T_{\rho}$, the tangent space of $G^{k}$ at $\rho$, spanned by $d f_{i}(\rho), d f_{2}(\rho), \ldots, d f_{w}(\rho)$ is of dimension $r_{1}$. It is obvious that $r=r_{1}$, (because $S$ is the locus described by $\left(R_{1}, R_{2}, \ldots, R_{L}\right)$ ). The following lemma is obvious. (See [2(2)].)

LEMMA. imbed $\operatorname{dim}$ of $S$ at $\rho=n-r_{1}$.

By this lemma and the above discussion we have the dimension of the kernel of $(d R)_{\rho}=$ imbed $\operatorname{dim}$ of $S$ at $\rho$. As a consequence of Lemma 1, we can now state our second lemma.

Lemma 2. For all $\rho \in S, \operatorname{dim}_{C} Z^{1}(Q, \operatorname{ad} \rho)=\operatorname{imbed} \operatorname{dim}$ of $S$ at $\rho$.

3. An analysis of the singularities in terms of the structure of the relators of $Q$. We are going to give a group theoretical determination of the regularity of the variety $S$ in terms of group cohomology. It is of convenience to introduce the Fox differential calculus on free groups to formulate our problem.

(1) Incidence matrices for a group. The group ring of $Q$ consists of all finite formal sums

$$
\sum n_{i} g_{i}, \quad n_{i} \in Z, g_{i} \in Q,
$$

with obvious addition and multiplication. Operation of $Q$ on any module $K$ induces naturally operations of $Z Q$ on $K$.

Let a sequence, namely $M$, of matrices with elements in $Z Q$ be constructed as follows:

$$
{ }^{1} M^{0}=\left(\begin{array}{c}
x_{1}-1 \\
x_{2}-1 \\
\vdots \\
x_{k}-1
\end{array}\right), \quad \text { a column matrix with }\left\{x_{1}, x_{2}, \ldots, x_{k}\right\}
$$

which are the set of generators of $Q$. 
(2) Given ${ }^{n} M^{n-1}$, let ${ }^{n+1} M^{n}$ be any matrix whose row space contains exactly those vectors $v$ such that $v \cdot{ }^{n} M^{n-1}=0$. This construction will be possible in general if we permit the number of rows of ${ }^{n} M^{n-1}$ to be infinite. If the number of rows of ${ }^{n} M^{n-1}$ is infinite, then we restrict the vectors in ${ }^{n+1} M^{n}$ to those with only a finite number of nonzero components.

If $K$ is any right $Q$-module, let $K_{n}$ be the $Q$-module of all vectors $v=\left(K_{1}, K_{2}, \ldots, K_{a_{n}}\right)$, where $a_{n}$ is the number of components from $K$, which is constructed as shown in (2). For $n>0, v \cdot{ }^{n} M^{n-1}$ lies in $K_{n-1}$; therefore one obtains a sequence of mappings,

$$
\rightarrow K_{n+1} \underset{{ }^{n+1} M^{n}}{\longrightarrow} K_{n} \underset{{ }^{n} M^{n-1}}{\longrightarrow} K_{n-1} \cdots K_{1} \rightarrow K_{0}=K .
$$

Since ${ }^{n+1} M \cdot{ }^{n} M^{n-1}=0$ identically in $Z Q$ by (ii), the image of each mapping is contained in the kernel of the next, and we may define the "homology groups"

$$
H_{n}(M, K)=\frac{\text { kernel of }{ }^{n} M^{n-1}}{\text { image of }{ }^{n+1} M^{n}}, \quad n>0 .
$$

For a left $Q$-module $K$, and $v$ in $K_{n}$, one has ${ }^{n+1} M^{n} \cdot v$ in $K_{n+1}$, and so an analogous sequence with arrows reversed

$$
\cdots \leftarrow K_{n+1} \stackrel{n+1 M^{n}}{\leftarrow} K_{n} \leftarrow \cdots \leftarrow K^{3} \stackrel{3 M^{2}}{\leftarrow} K^{2} \stackrel{{ }^{2} M^{1}}{\leftarrow} K^{1} \stackrel{M^{0}}{\leftarrow} K_{0}=K .
$$

One defines dually the "cohomology groups":

$$
H^{n}(M, K)=\frac{\text { kernel of }{ }^{n+1} M^{n}}{\text { image of }{ }^{n} M^{n-1}}, \quad n>0 .
$$

(Our description given here is very rough and somewhat vague; for reference to the above discussion one can consult [4].)

For our case to each representation $\rho$ of $Q$ into $G$, we can associate an adjoint representation ad $\rho$ of $Q$ into $\operatorname{End}(g)$; with respect to this adjoint representation one can regard $g$ as a $Q$-module. Therefore we can construct a sequence as before (the one describing the cohomology); in the sequel we shall call this sequence $(M)$.

Definition. $Q$ is of finite type iff $(M)$ is a finite resolution, i.e., each $K_{i}$ is of finite rank, and the sequence terminates somewhere for all $\rho \in S$.

We can define the Euler characteristic of $Q$ to be $X(Q)$,

$$
X(Q) \cdot \operatorname{dim}_{C} g=\sum_{i \geqslant 0}(-1)^{i} \operatorname{dim}_{C} H^{i}(Q, \operatorname{ad} \rho),
$$

which is an invariant independent of the choice of representations if $Q$ is of 
finite type. (For the proof one can consult [1].)

In the above formula $H^{i}(Q, \operatorname{ad} \rho)$ is the cohomology group with respect to the sequence $(M)$ associated to $\rho$.

(2) Relations between $\left({ }^{2} M^{1}\right)$ and the singularities of $S$. Consider a finitely presented group $Q=\left\{x_{1}, x_{2}, \ldots, x_{k}: R_{1}, R_{2}, \ldots, R_{L}\right\}$ as before. Associated to each $\rho \in S$, we can construct the sequence $(M)$ in the previous section.

Theoretically speaking we can determine $K^{i}$ and ${ }^{i+1} M^{i}$ for each $i$, but the most interesting fact is that $\left({ }^{1} M^{0}\right),\left({ }^{2} M^{1}\right), K^{0}, K^{1}, K^{2}$ can be determined in a particularly elegant form.

THEOREM. In the sequence $(M), K^{0}=g, K^{1}=g^{k}, K^{2}=g^{L}$ and,

$$
\left({ }^{1} M^{0}\right)=\left(\begin{array}{c}
x_{1}-1 \\
x_{2}-1 \\
\vdots \\
x_{k}-1
\end{array}\right)_{\mathrm{ad} \rho}, \quad\left({ }^{2} M^{1}\right)=\left(\frac{\partial R_{i}}{\partial x_{j}}\right)_{\mathrm{ad} \rho},
$$

where $\partial R_{i} / \partial x_{j}$ is the derivative of $R_{i}$ with respect to $x_{j}$ in terms of Fox differential calculus, and the subscripts simply mean to substitute ad $\rho\left(x_{i}\right)$ into the entries $\left\{x_{i}\right\}$ of the matrices.

(For the proof one can consult [4].)

An immediate conclusion from this theorem and Lemmas 1, 2 in $\S 2$ is:

LEMma 3. Kernel of $\left({ }^{2} M^{1}\right)=Z^{1}(Q, \operatorname{ad} \rho)=$ kernel of $(d R)_{\rho}$, all $\rho \in S$. Their dimensions are all equal to the imbedded dimension of $S$ at $\rho$.

$S$ can always be decomposed into many irreducible disjoint components, each component $S_{i}$ is irreducible and so is of pure dimension. In this section our discussion will only be restricted to one component of $S$, that is we are going to talk about irreducible variety only. Regarding $S$ as an imbedded analytic variety $G^{k}$, the imbedded dimension of $S$ at $\rho$ has been proved to be equal to $\operatorname{dim}_{C} Z^{1}(Q, \operatorname{ad} \rho)$ and $\operatorname{dim}_{C} \operatorname{ker}(d R)_{\rho}$ in the previous section. In general $\operatorname{dim}_{C} Z^{1}(Q$, ad $\rho)$ is greater than $\operatorname{dim}_{C} S$. However, the following fact follows immediately from the discussion in (2); $\rho \in S$ is regular iff $\operatorname{dim}_{C} Z^{1}(Q, \operatorname{ad} \rho)=\operatorname{dim}_{D} \operatorname{ker}(d R)_{\rho}=\operatorname{dim}_{C} S=h_{\rho}$ (the height of $S$ at $\rho$ ). It is of convenience to hold the following basic principle in mind (an easy consequence of Lemma 3):

Basic principle. If $S$ is irreducible, then $\rho \in S$ is regular iff dim of the kernel of $\left(\partial R_{i} / \partial x_{j}\right)_{\mathrm{ad} \rho}$ is the minimum of throughout all points of $S$. It is the same thing to say $\rho \in S$ is regular iff the rank of ${ }^{2} M^{1}$ of the sequence $(M)$ associated to $\rho$ maximizes throughout all points of the variety $S$.

This establishes the basic relation between ${ }^{2} M^{1}$ and the singularities of $S$. 
Therefore it is quite obvious that the singularities of $S$ depend mainly on the word structure of $R$.

Some concrete examples to demonstrate how to apply Fox differential calculus to treat the singularities of $S$ were carried in detail in the thesis of the author (Princeton, 1973).

4. Proof of the main theorem and corollary. The proof of our theorem is clear from the discussion of $\$ \S 2$ and 3 ; here we just go through the proof in detail as a matter of completion.

Let $Q=\left\{x_{1}, x_{2}, \ldots, x_{k}: R_{1}, R_{2}, \ldots, R_{L}\right\}$ be a finitely presented group of finite type. Then to each representation $\rho$ to $Q$ into $G$ we can associate an adjoint representation ad $\rho$ of $Q$ into $\operatorname{End}(g)$. Therefore we can construct a sequence $(M)$ as before. Since $Q$ is assumed to be a finite type, the sequence $(M)$ should terminate somewhere and each term of $(M)$ is of finite rank. We can define the Euler characteristic $X(Q)$ of $Q$ by the following formula:

$$
X(Q) \cdot \operatorname{dim} g=\sum_{i \geqslant 0}(-1)^{i} \operatorname{dim}\left(H^{i}(Q, \operatorname{ad} \rho)\right) .
$$

However,

$$
H^{1}(Q, \operatorname{ad} \rho)=\frac{\operatorname{kernel}\left({ }^{2} M^{1}\right)}{\operatorname{image}\left({ }^{1} M^{0}\right)}=\frac{Z^{1}(Q, \operatorname{ad} \rho)}{B^{1}(Q, \operatorname{ad} \rho)}
$$

it is clear that

$$
B^{1}(Q, \operatorname{ad} \rho)=\text { image of }\left({ }^{1} M^{0}\right), \quad Z^{1}(Q, \operatorname{ad} \rho)=\text { kernel of }\left({ }^{2} M^{1}\right) .
$$

By our Lemma 2, Lemma 3 in the previous section, $\operatorname{dim} Z^{1}(Q, \operatorname{ad} \rho)$ is just the imbedded dimension of $\rho \in S$; it had been pointed out before that $S$ is regular if and only if $\operatorname{dim}_{C} Z^{1}(Q, \operatorname{ad} \rho)=h_{\rho}$, the height of $\rho$ at $S$. Therefore we have

$$
\begin{aligned}
& \operatorname{dim} H^{0}(Q, \operatorname{ad} \rho)-\operatorname{dim} H^{1}(Q, \operatorname{ad} \rho)+\sum_{i \geqslant 2}(-1)^{i} \operatorname{dim} H^{i}(Q, \operatorname{ad} \rho) \\
& \quad=X(Q) \cdot \operatorname{dim} g
\end{aligned}
$$

and in general we have

$$
H^{0}(Q, \text { ad } \rho)=\text { kernel of }\left({ }^{1} M^{0}\right)
$$

and

$$
\operatorname{dim} H^{1}(Q, \operatorname{ad} \rho)=\operatorname{dim} Z^{1}(Q, \operatorname{ad} \rho)-\operatorname{dim} B^{1}(Q, \operatorname{ad} \rho)
$$

also 


$$
\operatorname{dim} H^{0}(Q, \operatorname{ad} \rho)+\operatorname{dim} B^{1}(Q, \operatorname{ad} \rho)=\operatorname{dim} g .
$$

Combining the above equalities we easily get

$$
\begin{gathered}
\operatorname{dim} g-\operatorname{dim} Z^{1}(Q, \operatorname{ad} \rho)+\sum_{i \geqslant 2}(-1)^{i} \operatorname{dim} H^{i}(Q, \operatorname{ad} \pi)=X(Q) \cdot \operatorname{dim} g, \\
\operatorname{dim} Z^{1}(Q, \operatorname{ad} \rho)+(X(Q)-1) \operatorname{dim} g=\sum_{i \geqslant 2}(-1)^{i} \operatorname{dim} H^{i}(Q, \operatorname{ad} \rho),
\end{gathered}
$$

and so for trivial reason we have

$$
\begin{aligned}
& \rho \in S \text { is regular if and only if } \\
& \sum_{i \geqslant 2}(-1)^{i} \operatorname{dim} H^{i}(Q, \operatorname{ad} \rho)=h_{\rho}+(X(Q)-1) \cdot \operatorname{dim} g,
\end{aligned}
$$

where $h_{\rho}$ is the height of the irreducible component of $S$ containing $\rho$. This gives the proof of Theorem (A).

For the proof of Theorem (B), it is completely trivial from the above discussion, as in general it is true

$$
\operatorname{dim} H^{0}(Q, \operatorname{ad} \rho)-\operatorname{dim} H^{1}(Q, \operatorname{ad} \rho)=\operatorname{dim} g-\operatorname{dim} Z^{1}(Q, \operatorname{ad} \rho)
$$

for any finitely presented group. Q.E.D.

In order to prove the corollary, we have to apply the identity theorem of Lyndon [4]. The main step is to show $\left({ }^{3} M^{2}\right)=0$ if $Q$ satisfies the conditions of our corollary in the introduction.

We quote the statement of Lyndon's identity theorem here without proof (see [4]).

R. C. LyNDON'S IDENTITY THEOREM. Let $F$ be the free group on generators $X_{1}, \ldots, X_{L+s}$; let $R_{1}, R_{2}, \ldots, R_{L}$ be cyclically reduced words in $F$ such that, for each $t, X_{t}$ and $X_{t+s}$ are the first and last (in the order of subscript) of the $X_{i}$ that occurs in $R_{t}$. Let $R_{t}=Q_{t}^{q_{t}}$ for $q_{t}$ maximal (i.e., $Q_{t}$ is primitive or irreducible); let $R$ be the smallest normal subgroup containing $R_{1}, R_{2}, \ldots, R_{L}$. If $\prod_{i=1}^{m} T_{i} R_{t_{i}}^{e_{i}} T_{i}^{-1}=1\left(T_{i}\right.$ in $\left.F, e_{i}= \pm 1, t_{i}=1, \ldots, L\right)$ then the indices 1, 2, $\ldots, m$ can be grouped into pairs $(i, j)$ such that $t_{i}=t_{j}, e_{i}=-e_{j}$ for certain integer $c_{i}, T_{i}=T_{j} Q_{i_{i}}^{c_{i}}(\bmod R)$.

Claim. $Q$ is of cohomology dimension two.

In order to prove this claim, the main step is to check $\left({ }^{3} M^{2}\right)$ is equal to zero in the sequence $(M)$ associated to the representation $\rho \in S$.

The following lemma, which can be found in [4], offers us a concrete method to obtain $\left({ }^{3} M^{2}\right)$ (also see the theorem in $\S 3(2)$ ).

LEMMA. With $Q$ as before, and if 


$$
P=\prod T_{i} R_{t_{i}}^{e_{i}} T_{i}^{-1} \text { is in }[R, R]
$$

then the following expression

$$
V_{t}=\sum_{t_{i}=t} e_{i} T_{i}, \quad t=1, \ldots, \rho_{2},
$$

defines a vector $V=\left(V_{1}, V_{2}, \ldots, V_{\rho_{2}}\right)$ in the row space of $\left({ }^{3} M^{2}\right)$, and every vector in the row space corresponds under (2) to some expression (1) for an element $P$ in $[R, R]$.

By the above lemma the vectors in the row space of $\left({ }^{3} M^{2}\right)$ are of the form $v_{t}=\sum e_{i} T_{i}$ corresponding to the identity $P=\prod T_{i} R_{t_{i}}^{e_{i}} T_{i}^{-1}=1$. (Note. $P$ $\in[R, R]$ is equivalent to, say, $P=1$ by Corollary 7.1, p. 659, of [4].) By the identity theorem the indices fall into pairs with $e_{i}=-e_{j}$ and $T_{i}$ $=T_{j} Q_{t_{i}}^{c_{i}}(\bmod R)$ for certain $c_{i}$. Hence $v_{t}$ can be written as a sum of the form $e_{i} T_{i}+e_{j} T_{j}=e_{i} T_{i}\left(Q_{t_{i}}^{c_{i}}-1\right)$. Therefore the entries $v_{t}$ of the vectors $v=\left(v_{t}\right)$ in the row space of $\left({ }^{3} M^{2}\right)$ are of the form $\sum u_{i}\left(Q_{t_{i}}-1\right)$ for some $u_{i}$ in $F$. However, by our assumption that the relators $R_{t_{i}}$ of the group are irreducible, that is $R_{t_{i}}=Q_{t_{i}}$, so that we may take ${ }^{3} M^{2}=0$ and so all the higher incidence matrices ${ }^{n+1} M^{n}$.

Applying our theorem, we have (note: all the $H^{i}(Q, \operatorname{ad} \rho)$, for $i \geqslant 3$, disappear)

$$
\rho \in S \text { is regular } \leftrightarrow \operatorname{dim} H^{2}(Q, \operatorname{ad} \rho)=h_{\rho}+(X(Q)-1) \cdot \operatorname{dim} g .
$$

It is an easy observation that $X(Q)=(1+L)-(L+s)=1-s$. This completes the whole proof.

The results of Riemann surface stated in the introduction as a remark follow easily from the duality of $H^{2}$ and $H^{0}$ of $Q$. As they are known results we shall not give the details here. (See the thesis of the author, Princeton, 1973.)

\section{REFERENCES}

1. Karl W. Gruenberg, Cohomological topics in group theory, Lecture Notes in Math., vol. 143, Springer-Verlag, Berlin and New York, 1970. MR 43 \#4923.

2. R. C. Gunning, (1) Lectures on vector bundles over Riemann surfaces, Math. Notes, Univ. of Tokyo Press, Tokyo; Princeton Univ. Press, Princeton, N.J., 1967. MR 37 \# 5888.

-, (2) Lectures on complex analytic varieties: The local parametrization theorem, Math. Notes, Univ. of Tokyo Press, Tokyo; Princeton Univ. Press, Princeton, N.J., 1970. MR 42 \# 7941. MR 45 \#2730.

(3) Local moduli for complex analytic vector bundles, Math. Ann. 195 (1971), 51-78.

, (4) Analytic structures on the space of flat vector bundles over a compact Riemann surface, Several Complex Variables, II, (Proc. Internat. Conf., Maryland, 1970), Lecture Notes in Math., vol. 185, Springer-Verlag, Berlin and New York, 1971, pp. 47-62. MR 49 \#3228.

3. H. Holmann, Quotienten Komplexer Räume, Math. Ann. 142 (1960/61), 407-440. MR 22, 11414. 
4. R. C. Lyndon, Cohomology theory of groups with a single defining relation, Ann. of Math. (2) 52 (1950), 650-665. MR 13, 819.

5. M. S. Narasimhan and C. S. Seshadri, Holomorphic vector bundles on a compact Riemann surface, Math. Ann. 155 (1964), 69-80. MR 29 \#4072.

6. Richard S. Palais, (1) On the existence of slices for actions of non-compact Lie groups, Ann. of Math. (2) 73 (1961), 295-323. MR 23 \# A3802. \#1664.

, (2) The classification of G-spaces, Mem. Amer. Math. Soc. No. 36, 1960. MR 31

7. K. Stein, (1) On factorization of holomorphic mappings, reprint from Proc. Conf. on Complex Analysis (Minneapolis, 1964), Springer-Verlag, Berlin and New York, 1965, pp. 1-7. MR 31 \#2419.

, (2) Maximale holomorphe und meromorphe Abbildungen. I, Amer. J. Math. 85 (1963), 298-315. MR 27 \#2651.

8. A. Weil, Remarks on the cohomology of groups, Ann. of Math. (2) 80 (1964), 149-157. MR 30 \# 199.

9. B. Wong, Thesis, Princeton Univ., 1973 (R. C. Gunning, superviser).

Department of Mathematics, University of Toronto, Toronto, Ontario, Canada M5S $1 \mathrm{Al}$ 